(Aus der Königl. Universitäts-Frauenklinik und der Königl. Hebammenschule. Director: Geheimrath Dr. Ritter v. Winckel, München.)

\title{
Drei Fälle von Kaiserschnitt an der Toten.
}

\author{
Von \\ Assistenzarzt Dr. E. von Seuffert.
}

F. von Winckel räth in seinem am 23. Januar 1892 im ärztlichen Bezirksverein München gehaltenen, in No. 5-d. Il. Jahrg. (1892) der "A erztlichen Rundschau" veröffentlichten Vortrag: „Der Kaiserschnitt an der Todten und an der Sterbenden "zur Publication jeder derartigen. Operation. Dieser Aufforderung glaube ich durch Bekanntgabe folgender von mir operirten Fälle entsprechen zu sollen.

1. Fa11: Am 10. Februar 1905, 8 Uhr, wurde die 24 J. alte I. p. Gärtnersfrau M. K. vom städtischen Krankenhaus l. d. I. in die Klinik eingeliefert mit der Diagnose "Meningitis tuberculosa“. Ausserdem war nur angegeben, dass Patientin seit ca. 36 Stunden komatös sei.

Defund: Mittelgrosse, ziemlich abgemagerte Frau. Komatöser Zustand. Manchmal reagirt Patientin ein wenig bei Berührung oder Anrufen. Athmung leicht stertorös. Puls ziemlich kräftig, regelmässig 109 Schläge pro Min., Pupillen gross. weit, reagiren auf Lichteinfall deutlich.

Leibesumfang $82 \mathrm{~cm}$. Fundus uteri in der Mitte zwischen Nabel und Herzgrube. Rücken des Kindes links. Kleine Theile rechts oben im Fundus. Köpfchen beweglich über dem Beckeneingang. In der linken Nabel-Spinal-Linie sind nicht immer, aber zeitweise sehr deutlich kindliche Herztöne zu hören. Frequenz 160-170. Uteruscontractionen nicht wahrnehmbar. Innere Untersuchung ergiebt: Scheide eng, Portio $2 \mathrm{~cm}$, wenig aufgelockert.

Aeusserer Mluttermund für die Fingerkuppe durchgängig, innerer Muttermund geschlossen.

Zwécks Einholung genauer Mittheilung über den bisherigen Verlauf der Krankheit und die Resultate etwaiger Untersuchungen (Ophthal- 
moskopie!) suchte ich alsbald mit dem behandelnden Arzt in telephonische Verbindung zu treten. Dieser war aber nicht erreichbar.

Da der Zustand der Patientin den Tod keineswegs als unmittelbar bevorstehend erscheinen liess, in Betreff der Diagnose lediglich eine kurze Mittheilung von anderer Seite vorlag, die augenblicklich nicht ergänzt werden konnte, kam natürlich ein sofortiger "Kaiserschnitt an der Sterbenden" nicht in Frage. Allerdings wurde die Möglichkeit, dass sich hierzu in Bälde die Indication ergeben könne, in Betracht gezogen. Besonderer diesbezüglicher Vorbereitungen bedurfte es nicht, da in der Klinik ja stets alles zur sofortigen Ausführung einer Köliotomie bereit ist.

Dagegen wurde die baldige Einleitung der künstlichen Frühgeburt, hauptsächlich im Interesse des lebenden und lebensfähigen Kindes projectirt, aber beschlossen, mit der Ausführung noch ein wenig zu warten, da der Zustand der Patientin offenbar durch den Transport beeinflusst war und sich scheinbar etwas besserte.

Kurz nach $1 / 212$ Uhr trat aber ganz plötzlich schwerer Collaps ein und als ich wenige Minuten später Patientin sah, war sie bereits fast pulslos, kindliche Herztöne trotz peinlichen Suchens nicht mehr zu hören. Um 11 Uhr $40 \mathrm{p}$. m. hatten Atmung uud Herzschlag vollkommen aufgehört. Mit einem Scalpell incidirte ich nun rasch Bauchdecken und Uterus. Eine arterielle Blutung trat hierbei nicht mehr ein. Beim Einreissen der sehr derben Eihäute lösten sich diese und die Placenta und wurden mit dem nicht ausgetragenen, apnoïschen Knaben entfernt. Die Wunde wurde vernäht. Durch Hautreize, warme Bäder und einige sehr vorsichtig ausgeführte Schultze'sche Schwingungen wurde das Kind rasch zum Athmen und bald auch zu leisem Schreien gebracht. Nach mehrstündigem Aufenthalt in dem von Winckel'schen Wärmebad hatte es sich soweit erholt, dass es in die inzwischen erwärmte Couveuse gebracht werden konnte.

Die nachträglich erhobene Anamnese ergab in Betreff der Mutter längeres Lungenleiden und schwerere Erscheinungen seit zwei Wochen. Der Vater des Kindes war angeblich gesund.

Die Section der Frau ergab: Sepsis. Tuberculöse, basilare Meningitis mit Abscessbildung. Gefässarrosion und Hämorrhagie im Bereich des hinteren, unteren Stirnlappens. Hämorrhagischer Hydrocephalus intern. Alter, hühnereigrosser, tuberculöser Herd der rechten Lungenspitze mit Verkalkung. Acute, miliare Tuberculose der Pleura mit disseminirter Einstreuung in allen Lungenabschnitten. Septischer Nilztumor. Trübe Schwellung der Leber und der Nieren.

Das Kind war $43 \mathrm{~cm}$ lang, $1780 \mathrm{~g}$ schwer. Die Kopfmaasse waren: Durchmesser: kleiner querer $7 \frac{1}{2}$, grosser querer $8 \frac{1}{2}$, gerader $10^{1} / 2$, grosser schräger $12^{1 / 2}$, kleiner schräger $91 / 2 \mathrm{~cm}$; Umfang $293 / 4 \mathrm{~cm}$. Placenta $400 \mathrm{~g}$. $13: 16 \mathrm{~cm}$. Länge der (nicht umschlungenen) Nabelschnur $30+7 \mathrm{~cm}$. An den Schultern und im Gesicht war die Haut noch stark mit Lanugo bedeckt. Keine deutliche inspiratorische Einziehung.

Aus dem Kinderjournal entnehme ich folgende Angaben: Am 13. 2. leichte, eitrige Secretion der noch wenig eingetrockneten Nabelschnur. Airolbepuderung. Beträchtlicher Icterus, der in den folgenden Tagen zunahm und bis zum 20. 2. vorhanden war. Erst am 21. 2. fiel der Nabelschnurrest $a b$, die Wunde heilte rasch. Bis zum 18. 2. nahm das Kind nur ganz wenig Nahrung aus der Flasche, wimmerte viel 
und nahm um $240 \mathrm{~g}$ ab. Vom 18.-21. 2. besserte sich die Nahrungsaufnahme und das Aussehen. Die Gewichtsabnahme dauerte aber noch fort bis zum 21. 2., da wog das Kind $1490 \mathrm{~g}$. Länge $42,2 \mathrm{~cm}$. Am 15. und 19. 2. sind leichte Durchfälle notirt. Bis zum 23. 2. war das Kind fast ununterbrochen in der Couveuse. Am 23. 2. erbot sich eine durch viele Milch belästigte Frau, das Kind mit dem Heberschlauch trinken zu lassen. Während der nächsten Tage wurde dem Kind ziemlich viel von dieser Ammenmilch zugeführt. Es erholte sich dabei sichtlich und nahm bis zum 3. 3. um $95 \mathrm{~g}$. zu. Am 1. 3. verliess die Amme die Klinik und eine neue Wärterin übernahm die Pflege des Kindes. Bei künstlicher Ernährung und Aufentbalt in der Couveuse trat am 5. 3. starker Durchfall und von da ab zunehmender Kräfteverfall ein. Gewicht am 7. 3. 1460 g. Am 9. 3. starb das Kind an Inanition. Gewicht $1420 \mathrm{~g}$. Länge $45 \mathrm{~cm}$. Die Section ergab ausser allgemeiner, hochgradiger Atrophie und leichter Anämie eine mässige Enteritis und offenbar terminale hypostatische Pneumonie beider Unterlappen.

Die mir in diesem Falle geglückte Entwickelung eines lebenden und zunächst am Leben gebliebenen Kindes durch Sectio post mortem ist nach einer Reihe von Autoren extrem selten:

Lange . . . . 141 Fälle; am Leben blieben 3 Kind.

\begin{tabular}{|c|c|c|c|c|c|}
\hline Schwarz & 107 & $"$ & , & $"$ &, \\
\hline Kehrer. & 61 & $"$ & $n$ & $"$ & 17 \\
\hline Horwitz u. Garazky & 379 & $"$ & $\because 9$ & $" n$ & , \\
\hline ohrn. & 90 & $"$ & & . & \\
\hline
\end{tabular}

Aber von Winckel hat schon 1892 in dem obengenannten Vortrag die Prognose für die Kinder weit besser gestellt und auch andere Statistiken aus neuerer Zeit geben bessere Resultate:

von Winckel. . 32 Fälle. Dauernd blieben am Leben $11 \mathrm{~K}$. Bauer . . . 15 " " " " $" 10$, Dicke (I.-D. Bonn

1903) • . $34 \quad " \quad, \quad, \quad$ ", 19,

Wenn auch nach F. Kleinhaus derartige Statistiken zu günstig lauten, da ja viele Fälle, in denen die Kinder todt extrahirt wurden, nicht bekannt gemacht sind, so werden sie, wie Fassbender gewiss mit Recht betont, dadurch ungünstig beeinflusst, dass so viele Fälle unreife Früchte betreffen. Bei fast ausgetragenen Kindern dürfte unter sonst günstigen Umständen die Prognose der Sectio post mortem nicht schlecht sein.

Mein Fall beweist übrigens gleich anderen, dass auch in prognostisch recht ungünstigen Fällen ein Kind lebend entwickelt und am Leben erhalten werden kann. Denn hier war: 
1. die Frucht erst 34 Wochen getragen,

2. die Mutter schon längere Zeit schwerkrank, ja seit 36 Stunden komatös,

3. Waren während der ganzen Agone keine Herztöne mehr gehört "worden,

4. lag laut Sectionsbericht eine septische Erkrankung vor.

Was 2. und 3. betrifft, hat schon von Winckel 1892 hervorgehoben, dass diese Umstände keineswegs die Prognose für das Kind aussichtslos machen. In seinen 12 an Herz- und LungenErkrankungen unterlegenen Fällen wurden nicht weniger als 10 Kinder gerettet. Neuere in von Winckel's Handbuch d. Gbh., III. Bd., I. T., S. 841, angeführte Fälle von Sommerbrodt, Everke u. A. stimmen hiermit überein. In dem von Winckel erwähnten Fall von Breslau (ebenfalls eine Lungenkranke) war keine Spur von kindlichen Herztönen zu hören, in dem von Dohrn (Eklampsie) 2 Stunden vor dem Tode kein Fötalpuls wahrnehmbar. Trotzdem wurden in beiden Fällen die Kinder lebend extrahirt.

Dass kindliche Herztöne nicht gehört werden können, kann übrigens auch sehr leicht durch Nebengeräusche bewirkt werden (Busalla, Centralblatt 1905, S. 1156), z. B. durch überlaute Athmung bei Lungenödem. Darum mussten wir auch in dem später zu besprechenden 2. Fall trotz Fehlens der kindlichen Herztöne die Hoffnung auf ein lebendes Kind keineswegs aufgeben.

Das Vorhandensein einer septischen Erkrankung endlich hätte nach Breslau die Prognose für das Kind geradezu hoffnungslos gemacht.

Von den in v. Winckel's Vortrag als prognostisch günstig bezeichneten Momenten sind für meinen Fall zu nennen: dass die Operation

1. fast unmittelbar nach Eintritt des Exitus letalis,

2. in der Klinik ausgeführt werden konnte.

Das Fehlen der in einer Klinik zur Verfügung stehenden sachverständigen Assistenz dürfte in dem zuletzt publicirten Fall von Nacke dem Kinde das Leben gekostet haben.

Schultze'sche Schwingungen, die dort eine tödtliche Leberblutung herbeigeführt haben, mussten in meinem 1. Fall zur Wiederbelebung auch angewandt werden. Dies geschah aber von 
mir und den diesbezüglich genau instruirten und geäbten Volontärärzten mit grösster Sorgfalt.

Bei der Prognose betreffend dauernde Erhaltung des zunächst geretteten kindlichen Lebens kommt in Betracht:

1. die Reife der Frucht,

2. die Ueberwindung von Schädigungen, die a) durch den Geburtsverlauf herbeigeführt wurden. Hier kommen, wenn der Geburtsakt schon begonnen hatte, alle bei gewöhnlichen Geburten vorkommenden schädlichen Einflüsse in Frage, sowie Folgen ungeeigneter Wiederbelebungsversuche, b) durch die Krankheit der Mutter verursacht sind. Solche sind aber nach ron Winckel in vielen Fällen nach sehr kurzer Zeit (34 Stunden: von Winckel's Vortrag: Fall von Hecker), jedenfalls aber nach ca. 14 Tagen überwunden. In meinem Fall bin ich geneigt, schon das schlechte Befindèn des : Kindes während der ersten 10 Tage mehr auf Rechnung der leichten Nabeleiterung und Enteritis zu setzen, als auf die der durch die Erkrankung der Mutter gesetzten Schädigungen. Nach 9 Tagen waren hier solche, wenn überhaupt vorhanden, sicher überwunden.

In den Statistiken der letzten Zeit und der Zukunft dürtten übrigens all' diese schädlichen Einflüsse eine weit weniger wichtige Rolle spielen als früher. Besitzen wir doch jetzt eine Reihe von Methoden zur erfolgreichen Bekämpfung der 'durch Frühgeburt, Asphyxie oder andere schädigende Momente herbeigeführten kindlichen Lebensschwäche. Durch richtig angewandte Schultze'sche Schwingungen, das von Winckel'sche Dauerbad, geeignete Wärmeschränke u. s. w. kann jetzt manches gefährdete Kind gerettet werden, das früher sicher verloren war. Die genannten 3 Wiederbelebungsmittel wurden in meinem Fall mit sichtbarem Erfolg angewandt. Das Gleiche gilt endlich auch

3. von der prognostisch so eminent wichtigen Art der Ernährung. Manches der zunächst durch Sectio post mortem geretteten Kinder, vielleicht auch das von unserem Fall, hätte durch längere Ammenernährung dauernd erhalten werden können. In unserem Fall war ihr günstiger Einfluss ganz unverkennbar. Gerade solchen Kindern dürften in Zukunft Wohlfahrtseinrichtungen wie die jetzt schon bestehenden Säuglingsheime zugute kommen, die auch Kindern Minderbemittelter Ammenernährung ermöglichen. Aber auch da, wo Frauenmilch nicht beschaffbar ist, ist die Pro- 
gnose weit besser geworden durch die enormen Fortschritte auf dem Gebiet der künstlichen Säuglingsernährung.

Die Frage der Prognose für dauernde Erhaltung des kindlichen Lebens hat freilich für die Sectio post mortem wenig Bedeutung. Umsomehr aber, wenn eine Sectio in agone in Betracht kommt.

2. Fall, Fr. U. T., 36 Jahr, VIIIp., Zimmermannsgattin, wurde am 9. März 1905, 10,30 Min. p.m. in vollkommen bewusstlosem Zustande eingeliefert. Anamnese (vom Gatten erhoben): Pat. war nie ernstlich krank. Keine Inesverdächtige Erkrankung bei ihr oder ihrem Gatten. In allen Schwangerschaften stark geschwollene Füsse. Die ersten 6 Entbindungen verliefen normal. Alle Kinder leben. Letzte Geburt Frühgeburt. Kind todt, Ursache unbekannt.

Heute (9. 3. 1905) Mittags plötzlicher Schwindelanfall mit folgender Lähmung der linken oberen und unteren Extremität. Das Sprachvermögen blieb bierbei erhalten. Bald darauf Anfall von Bewusstlosigkeit mit allgemeinen Krämpfen von 5 Minuten Dauer. Zustand nach denselben anverändert. $\operatorname{Lm} 3,5$ und 8 Uhr ähnliche Anfälle. Seit dem letzten besteht dauernd Bewusstlosigkeit. Das Kind soll leben.

Befund: Mittelgrosse, kräftige Person, vollkommen bewusstlos, hochgradiges Trachealrasseln. Mässige Cyanose. Puls 120, mittelkräftig. Zunge dick geschwollen, ragt aus dem Munde hervor, zeigt frische Bisswunden. Reflexe auch links erloschen, Pupillen mittelweit, starr. Geringe Oedeme beider Beine, Blase leer. Aeussere Untersuchung des Abdomens sehr erschwert durch die stürmische Athmung. Im Fundus rechts wahrscheinlich ein grosser Theil. Herztöne nicht $z u$ hören. Stand des Fundus 3 Querfinger unterhalb des Process. ensiform. Leibesumfang $86 \mathrm{~cm} . \quad 10,45$ Min. p.m. ergiebt die innerliche Untersuchung: Scheide weit, Portio erhalten, ca. $2 \mathrm{~cm}$ lang. Cervix für 1 Finger durchgängig. Blase steht. Vorliegt ein kleiner Theil, wahrscheinlich Fuss. Während dieser Untersuchung tritt hochgradige Cyanose ein. Der Puls wird fast unfühlbar. Die Athmung setzt aus. 3 Minuten später war die Herzthätigkeit vollkommen erloschen. Da kindliche Herztöne nicht gehört worden waren, wurde bis zu diesem Moment gewartet, dann mit einem Scalpell Bauchdecken und Uterus durch Längsschnitt in der Mittellinie eröffnet, wobei keine arterielle Blutung auftrat, aus dem Uterus jedoch ziemlich viel dickes, ganz dunkles Blut lief. Ich entwickelte rasch ein $45 \mathrm{~cm}$ langes, $1930 \mathrm{~g}$ schweres Mädchen. Das Kind war ganz starr, zeigte keinerlei Lebenszeichen und kounte nicht wieder belebt werden, war aber offenbar erst vor Kurzem gestorben (Nabelschnur ganz frisch). Die Placenta wurde noch entfernt $(400 \mathrm{~g})$, die Bauchdecken vernäht. Die anatomische Diagnose lautete: Ausgedehnte Hämorrhagie im vorderen Theil des Kleinhirns, des linken Schläfenlappens und des Centrum semiovale rechts. Hämatorn der weichen Hirnbäute. Gravidität mit Sectio caesarea post mortem. Hochgradiges Lungenoedem, parenchymatöse Nephritis.

Die Autopsie des Kindes ergab ausgedehnte Ecchymosen aller serösen Häute. Die Lungen waren vollkommen luftleer.

Sectio an der Sterbenden wäre in diesem Fall auch bei längerer Dauer der Agone wohl kaum in Betracht gekommen, die Vielgebärende hätte, noch dazu bei Fusslage, trotz der noch nicht erweiterten Cervix 
wohl rasch durch Dilatation per vias naturales entbunden werden können.

Interessant in diesem Fall war die Todtenstarre des Kindes. Ein Fall von intrauteriner Todtenstarre bei Sectio port mortem wurde zuerst 1886 von Dohrn publicirt und ein zweiter Fall von ihm 1895. In beiden Fällen war die Mutter eklamptisch. Der 3. derartige Fall, auch bei einer Eklamptischen, ist von Ludwig Seitz publicirt 1902.

In diesen Fällen zeigten allerdings die Kinder noch schwache Lebenszeichen, während solche in meinem Fall nicht mehr wahrnehmbar waren:

Eine ausführliche Literaturangabe über die bei Sectio post mortem und sonst beobachteten Fälle von Leichenstarre Neugeborener, wird, wie ich mit gütiger Erlaubniss des Autors schon jetzt bekannt gebe, in v. Winckel's Hdb. d. Geburtsh. III. Il. erscheinen, in dem von Prof. Stumpf bearbeiteten Abschnitt über forensische Geburtshülfe.

Das Vorkommen so sehr ausgedehnter Ecchymosen aller serösen Häute an Kindsleichen, bei denen die Sectio post mortem zu spät kam, wurde auch von $F$ üth in einem Fall beobachtet, den $\mathrm{Z}_{\mathrm{w}}$ eifel in einer Discussion erwähnt. Dort handelte es sich bei der Mutter auch um eine Gehirnerkrankung.

3. Fall: Am 27. 5. 1906, Abends 9 Uhr, wurde die 23 Jahre alte I p. Kellnerin $\mathrm{S}$. in bewusstlosem Zustand eingeliefert. Ihr sie begleitender Bräutigam machte folgende Angaben: Pat. war immer leicht erregbar und bekam häufig bei kleinen Aufregungen Anfälle, manchmal mit rasch vorübergehender Bewusstlosigkeit. Seit Beginn der Schwangerschaft litt Pat. an heftigen Kopfschmerzen, Schwindelanfällen und Erbrechen, besonders heftig waren diese Erscheinungen in letzter Zeit. Sie konnte nur sehr wenig Nabrung aufnehmen. S. magerte stark ab. Vor 3 Wochen consultirte Pat. einen Arzt, der ihren Harn untersuchte, angeblich aber nichts Pathologisches darin fand. Letzte Regel am 10. October.

Heute Abend 7 Uhr trat plötzlich ein Anfall von heftigem Zittern und Starrwerden der Augen, dann Starrkrampf und Bewusstlosigkeit auf. Angeblich kein Schäumen aus dem Mund oder Blauwerden der Haut.

Stalus praesens: Ziemlich kräftig gebaute Frau. Mittlerer Frnährungszustand. Etwas blasse Haut. Die bei Einlieferung vorhandene Bewusstlosigkeit liasst raseb nach. Pat. reagirt bald auf Anrufen und bewegt sich, zunächst allerdings nur schwach. Keine äusseren Verletzungen. Keine Zungenbisse, keine Oedeme. Temp. 36,60 , Puls 60. Fundus uteri handbreit über dem Nabel. Barchdecken sehr straff. Kindstheile sind zu fühlen, jedoch nicht sehr deutlich, offenbar viel Fruchtwasser. Kräftige kindliche Herztöne in der linken Nabelspinallinie am deutlichsten, aber auch ohue Unterbrechung und leiser bis 
oberhalb des Nabels zu hören. Die innere Untersuchung ergab: Scheide sehr eng. Portio noch mehrere Centimeter lang. Aeusserer $\mathrm{Mm}$. grübchenförmig. Cervix vollkommen geschlossen. Vorliegt beweglich über dem Eingang ein nicht genau zu erkennender Theil.

$\mathrm{Da}$ an verschiedenen Stellen kleine Theile zu fühlen waren, und kein entsprechend grosser Theil, da ferner an mehreren Stellen Herztöne gehört wurden und der Uterus für eine Gravidität von 33 Wocben ungewöhnlich gross war, schien das Vorhandensein mehrerer Früchte wahrscheinlich. Mittels Katheters honnten aus der Blase nur wenige Tropfen dunklen, leicht getrübten Harns entnommen werden, der nach starker Verdünnung mit Wasser noch deutlich positive Eiweissproben gab. Keine Wehen. Die Pat. kam allmählich wieder zu sich und gab bereits um 10 Chr p.m. auf jede Frage verständige Artwort. Sie klagte sehr über Schmerzen im Hinterkopf und im Kreuz. Zeitweise trat bei vollem Bewusstsein rasch vorübergebendes Zittern und leichtes Steifwerden der Extremitäten auf. Die Pupillen waren etwas erweitert. Die Reaction auf Lichteinfall etwas verlangsamt. Alle Reflexe ein wenig gesteigert. Auch war bei der Pat. eine gewisse Unruhe bemerkbar. 10 Uhr p. m. Temp. 36,90, Puls 66.

Das Vorbandensein einer Eklampsie war nach dem ganzen Befund sehr wahrscheinlicb, aber keineswegs sicher. Weder von uns noch von anderer Seite war ein typischer Anfall beobachtet worden. Oedeme fehlten, auch die Pulsverlangsamung war auffallend. Aber selbst, wenn wir die Diagnose für sicher gehalten hätten, war in diesem Fall zunächst Abwarten angezeigt. Die Anurie war das einzige schwere Symptom. Sonst deutete alles auf einen vorläufig noch leichteren Grad der Erkrankung hin und Patientin erholte sich zusehends. Die Frau war I para, Wehen waren nicht vorhanden, die Geburtswege noch vollkommen geschlossen. Eine sofortige Entbindung hätte also nur durch einen schweren Eingriff erzielt werden können. In Frage kam hier eigentlich nur vaginaler oder abdominaler Kaiserschnitt. Dazu kam, dass: die Prognose für die Frucht in der 33. Woche keine günstige war, besonders wenn es Mehrlinge waren.

Zur Anregung der Diurese erhielt nun Patientin feuchtwarme Einpackungen und reichlich heissen Tee; zur Beseitigung der vorhandenen Un'uhe, des Zitterns und der Kopfschmerzen $1,0 \mathrm{~g}$ Chloralhydrat! per rectum, um $10 \mathrm{Uhr}$ und $12 \mathrm{Uhr}$ p. m. Während der Nacht schlummerte Patientin zeitweise, klagte aber viel über Schmerzen im Hinterkopf und im Rückẹn. Das Zittern der Extremitäten liess nach. Uteruscontractionen waren nicht bemerkbar, die kindlichen Herztöne dauernd gut und kräftig. Kein Fieber. Puls regelmässig, ziemlich kräftig, etwas verlangsamt. Harn konnte jedoch nur tropfenweise entleert werden: Um 4 Uhr 45 Minuten a. m. trat plötzlich ein Anfall mit Strecken der Extremitäten, vollkommener Bewusstlosigkeit, rasselnder Athmung und sehr frequentem, gespanntem Puls auf. Keine Cyanose. Verengerung des Pupillen. Der Anfall daverte mehrere Minuten. Das Bewusstsein kehrte nach 20 Minuten, jedoch unvollkommen zurïck. Patientin griff dann oft stöhnend nach dem Hinterkopf.

Ein gleichzeitig unsere Hilfe in Anspruch nehmender anderer schwerer Fall machte leider eine ganz genaue Beobachtung dieses Anfalls und sofortiges Eingreifen unmöglich. Die Diagnose Eklampsie schien aber nunmehr gesichert und die vaginale Sectio caesarea wäre baldmöglichst ansgeführt worden. Indessen ebe dies geschehen konnte, 
trat plötzlich hochgradige Cyanose, Streckkrampf, Aussetzung der Athmung und 5 Minuten später Exitus letalis ein. Während dieser 5 Minuten waren zwischen Nabel und Symphyse anfangs noch recht kräftige, allmählich langsamer werdende kindliche Herztöne zu hören. Noch während der Agone die Sectio caes. lege artis, vorzunehmen, war uns nicht möglich. Ich wartete daher, bis der Exitus zweifellos eingetreten war und entwickelte dann durch Medianschnitte Zwillinge. Bei beiden Knaben war der Herzschlag deutlich, wenn auch verlangsamt zu hören (ca. 80.p. M.) und einzelne Inspirationen wahrnehmbar. Trotz energischer Wiederbelebungsversuche konnte jedoch keines zu eigentlichem Schreien gebracht werden und nach 30 Minuten war bei dem kleineren, zuerst entwickelten Kinde, nach 40 Minuten auch bei dem anderen die Herzthätigkeit vollkommen erloschen.

Beide Knaben waren $43 \mathrm{~cm}$ lang. Der zuerst entwickelte wog 1610 g; der zweite 1640 g. Die vollkommen getrennten Placenten wogen $390 \mathrm{~g}$ und $400 \mathrm{~g}$. Das in grosser Menge abgeflossene Fruchtwasser war ohne Meconium. Post mortem konnte mit Katheter $50 \mathrm{~g}$ Urin entnommen werden. Da sich in diesem 3 pll. Albumen sowie hyaline und granulirte Cylinder fanden, war uns die Diagnose Eklampsie zweifellos.

Die Section ergab aber: Gliosarkom des rechten Hinterhauptlappens. Allgemeine Anämie. Geringe Dilatation und Hypertrophie des Herzens. Trübe Sehwellung beider Nieren. Verkäsende Tubereulose der Hilusdrüsen. Die Section der Früchte ergab nichts Besonderes. $\mathrm{Zu}$ dieser Diagnose konnten wir bei der Kürze der Beobachtungszeit nicht kommen. Wäre es aber möglich gewesen, so wäre hier, vorausgesetzt, dass man nicht prinzipieller Gegner ist, gewiss eine Sectio caesarea in moritura indicirt gewesen, durch die wahrscheinlich die Kinder, zunächst wenigstens, wären gerettet worden. Sehr schwer, wie in den meisten Fällen, wäre es allerdings gewesen, den Zeitpunkt zu bestimmen, in welchem man zu dieser Operation berechtigt war, wenn mall mit Runge und anderen diese Operation nur ausführen will, wenn der Tod innerhalb 12 Stunden als sicher vorauszusehen ist. Und die von Runge aufgestellte, allgemein anerkannte Forderung, die Sectio an der noch lebenden Frau unter allen Umständen lege artis auszuführen, verbot auch bei beginnenden agonalen Erscheinungen undesificirt zu operiren und so die den Früchten hier wahrscheinlich verhängnisvoll gewordenen, letzten 5 Minuten zu sparen.

Die von uns auf Grund einer falschen Diagnose geplante Operation wäre natürlich nicht als Sectio in moritura, sondern zur Therapie der Eklampsie ausgeführt worden.

Als schonendste, rascheste und technisch relativ einfache Entbindungsart wird die abdominale Sectio heute nicht nur bei Eklampsie, sondern auch bei anderen, das mütterliche und kindliche Leben augenblicklich bedrohenden Zuständen von vielen als berechtigt, von manchen geradezu als indicirt betrachtet.

In Folge dessen wird die Frage einer Sectio in moritura lediglich zur Rettung des Kindes unter Hintansetzung des vielleicht 
doch nicht verlorenen mütterlichen Lebens in Zukunft viel seltener an den Arzt herantreten.

Gerade da, wo, wie die Fälle von Runge und Poten zeigen, die Entscheidung, ob man wirklich eine Sterbende vor sich hat (Eklampsie, schwere Herzerkrankung) besonders schwer ist, kann jetzt die Sectio, lege artis natürlich, ruhig ausgeführt werden, in der Hoffnung, durch sie vielleicht auch noch die Mutter retten zu können.

\section{Benutzte Literatur.}

Bauer, Sectio caesarea post. mort. Monatsschr. f. Geb. u. Gyn. Bd. 16. S. 811. Breslau, Monatsschr. f. Geb. u. Fr. 1868. Bd. 24. S. $81 \mathrm{ff}$.

Busalla, Centralbl. f. Gyn. 1905. S. 1153. Ueber den Kaiserschnitt in der Agone und post morten bei Herzfehlerliranken.

Dohrn, Zwei Fälle aus der Klinik. I. Kaiserschnitt nach dem Tode der Mutter. Centralbl. 1886. S. 113.

Derselbe, Tonische Muckelcontractur bei todtgeborenem, frühzeitigem Kind einer Eklamptischen. Sectio caesarea post mort. Centralbl. 1895. S. 45.

Dohrn, Der Kaiserschnitt an verstorbenen Schwangeren. Volkmann's Sammlung klin. Vortr. N. F. No. 188. 1897.

H. Fassbender, Berlin, Geschichte der Geburtshülfe. 1906. S. 979 ff.

Fehling's Artikel in P. Müller's Handbuch d. Geb. 1889. Bd. Ill. S. 211. Füth, Centralbl. f. Gyn. 1905. No. 23. Ueber Sectio caesarea an der Moribunden.

Kehrer, Zur Kaiserschnittfrage. Archiv f. Geb. u. Gyn. Bd. 27. S. 2.

F. Kleinhaus, Prag, Der Kaiserschnitt an der Sterbenden und Todten in von Winckel's Handb. d. Geb. Bd. IIl, 1. S. $838 \mathrm{ff}$.

Lange, Kritische und statistische Bemerkungen zur Lehre vom Kaiserschnitt an der Todten. Casper's Wochenschrift. S. $112 \mathrm{ff}$.

W. Nacke, Berlin, Ueber den Kaiserschnitt an der Todten bei Herzkranken. Centralbl. 1906. No. 10. S. 302.

Poten, Centralbi. f. Gyn. 1905. S. 2153.

Runge, Zeitschr. f. Geb. u. Gyn. 1883. Bd. IX. S. 2. Die Berechtigung des Kaiserschnittes an der Sterbenden und der mit ihm concurrirenden Entbindungsvorfahren.

Schwarz, Der Kaiserschnitt an der Todten. Monatsschr. f. Geb. n. Fr. 1862. S. 173.

F. von WinckeI, Der Kaiserschnitt an der Todten und an der Sterbenden. Vortrag, gehalten im ärztl. Bezirksverein München am 23. Januar 1892. Enthalten in No. 5 der Aerztlichen Rundschau. 1892.

Zweifel, Discussion zu Füth's Vortrag. Centralbl. f. Gyn. 1905. S. 726. 